\title{
Polypoidal choroidal vasculopathy in pachychoroid: combined treatment with photodynamic therapy and aflibercept
}

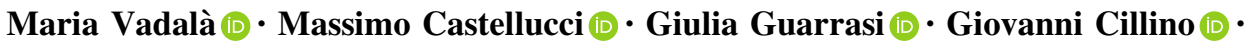

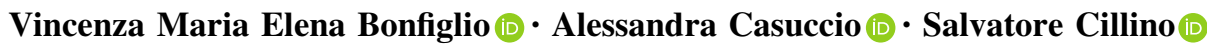

Received: 9 January 2021 / Accepted: 22 September 2021

(C) The Author(s), under exclusive licence to Springer Nature B.V. 2021

\begin{abstract}
Introduction To evaluate the effects of combined therapy using intravitreal Aflibercept (IVA) and photodynamic therapy (PDT) on polypoidal choroidal vasculopathy related to pachychoroid disease (PPCV). Methods Patients with PPCV were treated with PDT combined with 3 IVA injections on a monthly basis, followed by pro re nata criteria. The 12-month followup consisted of multiple revaluations of visual acuity and SS-OCT parameters of clinical activity.

Results Nineteen eyes were included in the study; mean age was 65.5 years. Visual acuity improved
\end{abstract}

Maria Vadalà and Massimo Castellucci have contributed equally to this work.

Data were partially presented as an oral poster presentation at EURETINA Congress, Paris 6th September 2019.

M. Vadalà $(\bowtie) \cdot$ M. Castellucci $(\bowtie)$.

G. Guarrasi - G. Cillino - V. M. E. Bonfiglio - S. Cillino

Biomedicine, Neuroscience and Advance Diagnostic

Department, Ophthalmology Institute, University of

Palermo, Villa Belmonte, Via Cardinale Rampolla 1,

90142 Palermo, Italy

e-mail: maria.vadala@unipa.it

M. Castellucci

e-mail: maria.vadala@unipa.it

\section{A. Casuccio}

Department of Health Promotion, Internal Medicine and of Excellence, University of Palermo, Mother-Child Care, Palermo, Italy after 12 months $(0.35 \pm 0.25$ to $0.2 \pm 0.20 \log \mathrm{MAR}$, $\mathrm{p}=0.005)$. Percentage of eyes with intraretinal and subretinal fluid reduced from baseline to the 12-month follow-up (from 52.6 to $10.5 \%, p=0.12$, and from 89.5 to $5.3 \% p=0.0009$, respectively). Central retinal and mean macular thicknesses reduced $(258 \pm 39.6$ to $204.8 \pm 38.8 \mu \mathrm{m} \quad p=0.04$ and $293.8 \pm 32.1$ to $248.1 \pm 29.6 \mu \mathrm{m} p=0.017$, respectively). Central choroidal and mean choroidal thicknesses also displayed a reduction $(328.6 \pm 54.9$ to $289.8 \pm 44.6 \mu \mathrm{m}$ $p=0.001$ and $314.5 \pm 55.3$ to $287.9 \pm 47.6 \mu \mathrm{m}$ $p=0.015$, respectively). The mean number of injections was 4.6/year.

Conclusion The results support the use of a combined therapy with Aflibercept and PDT in PPCV. This treatment would act in synergy, with anti-VEGF controlling exudation and PDT closing the aneurysmal vessel and reducing choroidal congestion.

Keywords Aflibercept · Pachychoroid .

Photodynamic therapy · Polypoidal choroidal vasculopathy $\cdot$ Swept Source OCT

\section{Introduction}

Pachychoroid (pachi-[prefix]: thick) describes an anomalous and definitive thickening of the choroid; 
it is assumed to be thicker than $300 \mu \mathrm{m}$, while the accepted normal range is between 191 and $350 \mu \mathrm{m}$ [1].

The definition of pachychoroid includes several clinical features such as a thicker choroid, dilated and straight choroidal vessels that end abruptly, choroidal hyperpermeability on ICGA, compression of Sattler's layer due to dilated choroidal vessels in Haller's layer and choriocapillaris thinning visible on OCT, RPE changes and atrophy, and serous RPE detachment [2-5].The hyperpermeability of choriocapillaris and pachyvessels causes fluid and lipoproteins to move toward the choroidal stroma. While eyes with thicker choroid do not always display choroidal hyperpermeability, eyes with hyperpermeability phenomena are always characterized by pachychoroid [6]. Choroidal neovascularization has also been described here [7]. Such findings define pachychoroid even if choroidal thickness is within normal limits $[3,7]$.

The pachychoroid disease spectrum includes several clinical entities: central serous chorioretinopathy (CSC), pachychoroid pigment epitheliopathy (PPE), neovascularization in pachychoroid (PNV), pachychoroid-related polypoidal choroidal vasculopathy (PPCV), focal choroidal excavation (FCE) and peripapillary pachychoroidal syndrome (PPS). The overlapping and concomitance of conditions are described as well as the progression or evolution from one manifestation to another, supporting the hypothesis of a common pathogenesis [7, 8].

Patients with central exudative complications such as CSC, PNV and PPCV with macular fluid are in need of treatment [7]. Classic polypoidal choroidal vasculopathy (PCV) treatment is currently still controversial even though intravitreal anti-VEGF drugs are the suggested option; thermal laser, photodynamic therapy with verteporfin (vPDT), subthreshold laser therapy or a combination of these treatments are also considered [7]. However, PCV is probably the same clinical feature of different pathogenic processes, when associated with pachychoroid or with agerelated macular degeneration (AMD) [9, 10]. The management of PPCV is under ongoing review as few studies have examined this subtype of lesion $[10,11]$. We examined visual and morphological changes in a cohort of naive patients affected by PPCV and treated with a combination of vPDT and intravitreal injections of Aflibercept (IVA), based on the pathogenetic hypothesis of this peculiar neovascularization.

\section{Materials and methods}

This is a prospective and consecutive uncontrolled case series study which evaluates a combined treatment with vPDT and Aflibercept in naive patients affected by macular PPCV. All patients were Caucasian, recruited between January and October 2017 at the Ophthalmology Clinic of the University of Palermo (Italy). We thoroughly informed all patients about the procedures and written informed consent documents were signed and approved by the Ethics Committee of the University of Palermo n. 8/2017 in compliance with the tenets of the Declaration of Helsinki.

The diagnosis of pachychoroid disease was made considering typical characteristics, such as reduced fundus tessellation on color fundus photographs, pathologically dilated outer choroidal vessels on OCT (dilated Haller vessels with attenuation or compression of Sattler's layer and choriocapillaris) and ICGA (straight, dilated and hyperpermeable choroidal vessels within the macular area) images, and no typical sign of AMD. FAF was performed to find areas of hyper-AF or hypo-AF and gravitational descending tracts as signs of pre-existing CSC. The diagnosis of exudative macular PPCV was based on Swept Source Optical Coherence Tomography (SSOCT) and fundus autofluorescence (FAF) (Triton FAPlus, Topcon, Japan), Fluorescence Angiography (FA) and Indocyanine Green Angiography (ICGA) images (TRC 50IX, Topcon, Japan); clinical criteria were presence of pachychoroid as defined before, presence of elevated orange-red nodular choroidal macular lesion at fundus observation and presence of $\mathrm{CNV}$ aneurysms/polyps/vascular networks in the posterior pole on FA/ICGA as defined by the EVEREST I/II study group [12].

Two independent examiners (MC and GG) evaluated OCT, FA and ICGA images in order to reach an agreement on the diagnosis; if there was a disagreement, the diagnosis was determined by a third examiner (MV).

The exclusion criteria included any coexisting ophthalmic diseases if they were not related to pachychoroid (i.e., glaucoma, uveitis, axial length $>$ $25 \mathrm{~mm}$, aphakia, histories of retinal vessel occlusion, diabetic retinopathy, retinal detachment), previous intravitreal treatments or cardiovascular events with anti-VEGF contraindications, laser photocoagulation, 
and previous ocular surgery other than phacoemulsification.

Comprehensive ocular examinations, including Snellen corrected distance visual acuity (CDVA), slit-lamp biomicroscopy with fundus examination and IOP evaluation, SS-OCT imaging (3D $7 \times 7 \mathrm{H}$ and radial 6.0 macular scan protocols), FAF, FA and ICGA were carried out on all patients at baseline conditions. For the purpose of this study, the following outcomes were evaluated: Snellen CDVA converted to the logarithm of the minimal angle of resolution (logMAR) for statistical analysis; disease activity reported as a percentage of cases of intraretinal (IRF) or subretinal fluid (SRF) presence; central retinal thickness (CRT) and central choroidal thickness (CCT) considered as the central $1 \mathrm{~mm}$ zone of the ETDRS grid, from Inner Limiting Membrane (ILM) to Outer Layer of Retinal Pigmented Epithelium (RPE) and from Bruch's Membrane (BM) and outer choroideal border respectively, as measured automatically by means of Topcon IMAGEnet 6 software tool, version 10.1.5; mean macular thickness (MMT) and mean choroidal thickness (MCT) measured in the central $6 \mathrm{~mm}$ zone as described above; mean number of IVA. Greatest linear dimension of neovascular lesion was manually measured by means of a Topcon IMAGEnet 6 software tool, version 10.1.5. All patients were treated with consecutive 3 monthly IVAs $(2.0 \mathrm{mg}$ of Aflibercept in $0.05 \mathrm{ml}$ ) and standard full-fluence vPDT with verteporfin performed a week after the first IVA. IVA was performed in operating theatre, using a sterile eye drape and eyelid speculum, oxybuprocaine hydrochloride eyedrops and 5\% for topical anesthesia and aqueous povidone iodine solution to clean and disinfect the eye to be injected. Aflibercept was injected in inferotemporal quadrant at $4.0 \mathrm{~mm}$ from limbus in phakic eyes and at $3.5 \mathrm{~mm}$ from limbus in pseudophakic eyes. Topical antibiotic drops 4 times a day was prescribed for 5 days after the injection. The Verteporfin dose for PDT was BMIbased $\left(6 \mathrm{mg} / \mathrm{m}^{2}\right)$ and photodynamic laser light at $689 \mathrm{~nm}$ delivered $50 \mathrm{~J} / \mathrm{cm}^{2}$ at an intensity of $600 \mathrm{~mW} /$ $\mathrm{cm}^{2}$ over $83 \mathrm{~s}$ on the macular area, setting the spot LASER size as the greatest linear dimension of the neovascular lesion plus $1000 \mu \mathrm{m}$ on ICGA image. The visual and anatomical outcomes were evaluated on a monthly basis; for the purpose of this study results from the months $1,3,4.5,6$, and 12 are reported. FA and ICGA were performed when necessary on clinical judgment. No additional treatment was performed during follow-up unless signs of neovascular activity were detected, such as intraretinal or subretinal fluid and subretinal hemorrhages. In those cases, we decided to treat patients as PRN (pro re nata, as needed) protocol with Aflibercept.

\section{Statistical analysis}

Statistical analysis of quantitative data, including descriptive statistics, was performed on all components. The normality distribution of continuous variables was assessed by the Shapiro-Wilk test. All the continuous data are expressed as a mean \pm standard deviation and range. CDVA results are presented as a logarithm of the minimal angle of resolution (LogMAR) units. The univariate analysis of variance (ANOVA test) for repeated measures with post-hoc analysis was used for parametric analysis, while for nonparametric data analysis the Friedman test was carried out. Interobserved variability was analyzed using Cohen's kappa. The data were analyzed by Epi Info software (version 6.0; Centre for Disease Control and Prevention, Atlanta, GA) and IBM SPSS Software version 21.0 (SPSS, Inc., Chicago, IL). All p values were two-sided, and $\mathrm{p}$ values less than 0.05 were considered statistically significant.

\section{Results}

Patient characteristics

Nineteen eyes of eighteen patients were included in the study of which fourteen patients were men and four were women. The mean age of the patients was 65.5 years $( \pm 8.3$, range $52-80)$.

Mean number of IVA was 4.6/year (range: 3-7 IVA/year); 3 eyes (16\%) required 7 IVA/year as suggested by Aflibercept protocol, 6 eyes (32\%) underwent 2 additional IVA, 3 eyes (16\%) 1 additional IVA after loading dose.

Table 1 shows the clinical data starting at baseline up to the end of the 12-month follow-up. Figures 1 and 2 show multimodal imaging of one paradigmatic case of the series. 
Table 1 Clinical data

\begin{tabular}{lllllll}
\hline & Baseline & 1 mo & 3 mo & 4.5 mo & 6 mo & 12 mo \\
\hline $\begin{array}{l}\text { Mean } \pm \text { SD, range } \\
\text { CDVA (logMAR } \pm \mathrm{SD},\end{array}$ & $0.35 \pm 0.25$ & $0.28 \pm 0.24$ & $0.26 \pm 0.26$ & $0.26 \pm 0.25$ & $0.23 \pm 0.23^{*}$ & $0.2 \pm 0.2^{* *}$ \\
range $)$ & $(0.1-1)$ & $(0-1)$ & $(0-1)$ & $(0-1)$ & $(0-0.8)$ & $(0-0.7)$ \\
IRF $(\%)$ & $52.6 \%$ & $26.3 \%$ & $36.8 \%$ & $36.8 \%$ & $15.8 \%$ & $10.5 \%$ \\
SRF $(\%)$ & $89.5 \%$ & $31.6 \% *$ & $15.8 \% * *$ & $15.8 \% * *$ & $10.5 \% * *$ & $5.3 \% * *$ \\
CRT $(\mu \mathrm{m} \pm \mathrm{SD}$, range) & $258 \pm 39.6$ & $210.9 \pm 36.5$ & $211.7 \pm 52.4$ & $202.2 \pm 38.7^{*}$ & $199.8 \pm 28^{*}$ & $204.8 \pm 38.8^{*}$ \\
& $(203-372)$ & $(129-285)$ & $(140-339)$ & $(139-299)$ & $(141-245)$ & $(130-299)$ \\
MMT $(\mu \mathrm{m} \pm \mathrm{SD}$, range $)$ & $293.8 \pm 32.1$ & $253.3 \pm 25.9^{*}$ & $248.8 \pm 27.4^{*}$ & $241 \pm 24.8^{*}$ & $239.6 \pm 25.6^{* *}$ & $248.1 \pm 29.6^{*}$ \\
& $(251-393)$ & $(212-303)$ & $(201-297)$ & $(201-291)$ & $(212-299)$ & $(215-313)$ \\
CCT $(\mu \mathrm{m} \pm \mathrm{SD}$, range $)$ & $328.6 \pm 54.9$ & $290.8 \pm 55.9^{* *}$ & $294.4 \pm 56.1 * *$ & $291.8 \pm 53^{*}$ & $293 \pm 51.7 *$ & $289.8 \pm 44.6^{* *}$ \\
& $(251-425)$ & $(198-376)$ & $(199-407)$ & $(200-372)$ & $(211-377)$ & $(214-378)$ \\
MCT $(\mu \mathrm{m} \pm \mathrm{SD}$, range $)$ & $314.5 \pm 55.3$ & $281.6 \pm 51^{* *}$ & $286.3 \pm 58.8^{* *}$ & $289.6 \pm 54.6^{*}$ & $294.9 \pm 54.7^{*}$ & $287.9 \pm 47.6^{*}$ \\
& $(207-403)$ & $(197-379)$ & $(188-405)$ & $(186-378)$ & $(195-381)$ & $(205-380)$ \\
\hline
\end{tabular}

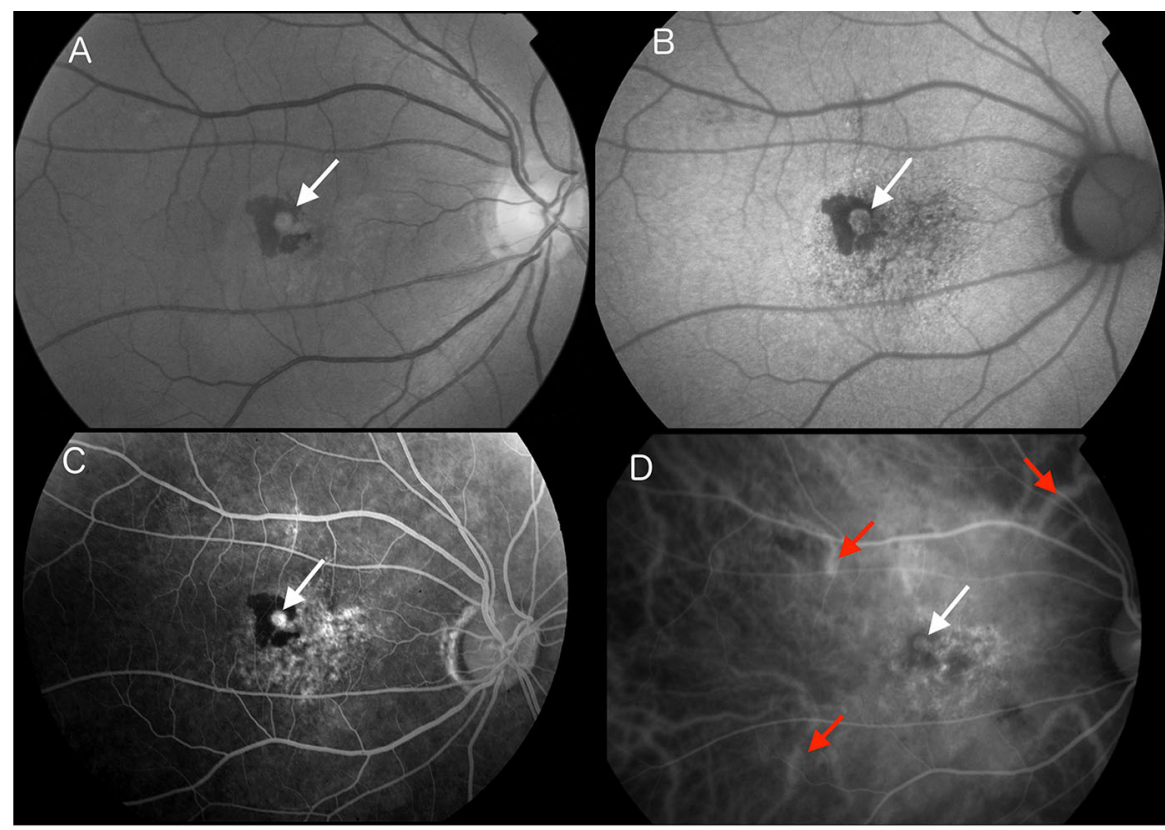

Fig. 1 Multimodal imaging of a 54-year-old male patient affected by PPCV in right eye at initial presentation. White arrow indicates polypoidal lesion. a Near-infrared fundus image showing macular neovascular lesion surrounded by intraretinal hemorrhage. b FAF showing speckled gravitational ipofluorescence, sign of pre-existing CSC. cFA showing macular hyperfluorescence indicative of leakage secondary to an occult choroidal neovascularization, surrounded by fluorescence block due and characteristic pigment epitheliopathy hyperfluorescence. d Early phase ICGA showing hyperfluorescent macular lesion and large caliber choroidal vessels (red arrows) 


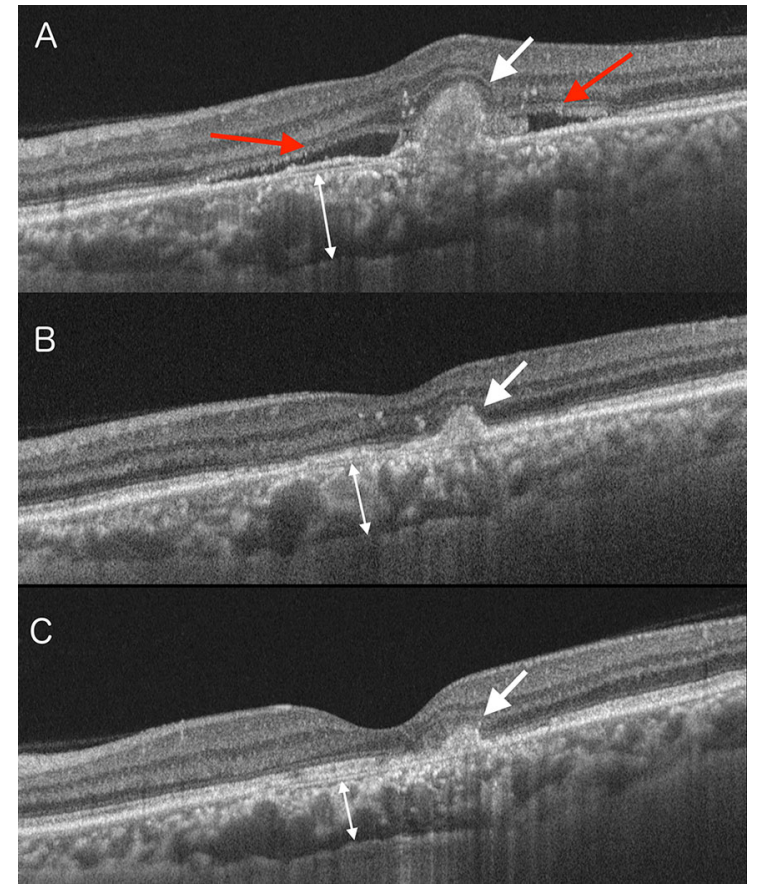

Fig. 2 Sequential SS-OCT imaging in the right eye of the patient of Fig. 1, showing PPCV response to combined treatment during 12-months follow-up. a Baseline imaging showing PPCV characteristics: polypoidal lesion (white arrow) as mild hyperreflective macular lesion surrounded by subretinal fluid (red arrows), thick choroid (double-arrow white line). b 4.5 months imaging: PPCV already treated with 3 IVA monthly + PDT. No evidence of neovascular lesion activity reported, as PPCV showed a reduction in size (white arrow). c 12-month imaging: no recurrences after combined treatment protocol, hypereflective lesion is further reduced in size (white arrow), still without fluid. Choroidal thickness also reduced (double-arrow white line)

\section{CDVA outcomes}

CDVA improved from baseline after 1, 3, 4.5 and 6 months with statistical significance being observed at 6 and 12 months (from $0.35 \pm 0.25$ to $0.28 \pm 0.24$ $p=0.17,0.26 \pm 0.26 \mathrm{p}=0.11,0.26 \pm 0.25 p=$ $0.08,0.23 \pm 0.23 p=0.04,0.2 \pm 0.2 p=0.005$, respectively) as shown in Fig. 3. After 12 months, all eyes but one improved from their respective baselines.

\section{Disease activity}

The percentage of patients with IRF changed with no statistical significance at 1, 3, 4.5, 6 and 12 months (from 52.6 to $26.3 \%, p=0.45,36.8 \% p=0.8,36.8 \%$

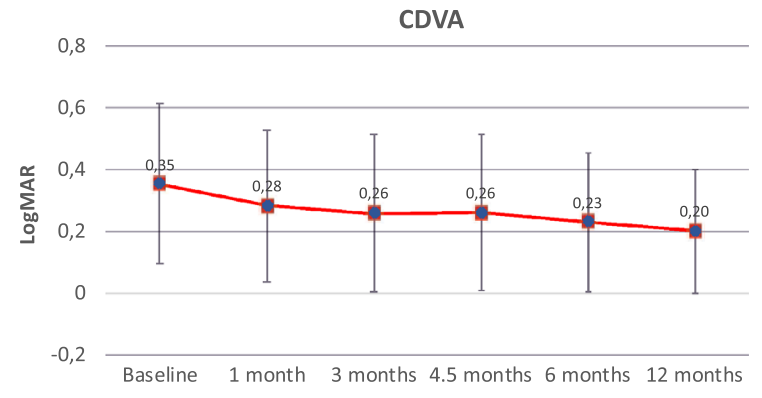

Fig. 3 Graphic of clinical data: visual acuity, CDVA = Corrected Distance Visual Acuity; LogMAR $=$ logarithm of the minimum angle of resolution, $* p \leq 0.05$, * $p \leq 0.005$ compared with baseline

$p=0.8, \quad 15.8 \% p=0.2,10.5 \% p=0.12$, respectively); such results are shown in Fig. 4.

The percentage of patients with SRF statistically reduced after 1, 3, 4.5, 6 and 12 months (from 89.5 to $31.6 \%, p=0.03,15.8 \% p=0.004,15.8 \% p=0.004$, $10.5 \% p=0.002)$. After 12 months residual SRF was present in $5.3 \%(p=0.0009)$ of cases (shown in Fig. 4).

\section{OCT measurements}

There was a high interobserver reproducibility for SSOCT analysis ( $\mathrm{k}=0.7403,95 \% \mathrm{CI}: 0.70-0.86)$.

CRT showed progressive reduction after 1, 3, 4.5, 6 and 12 months compared to baseline as shown in Fig. 5 (from $258 \pm 39.6 \mu \mathrm{m}$ to $210.9 \pm 36.5 \mu \mathrm{m}$ $p=0.09,211.7 \pm 52.4 \mu \mathrm{m} p=0.28,202.2 \pm 38.7$ $\mu \mathrm{m} p=0.03,199.8 \pm 28 \mu \mathrm{m} p=0.007,204.8 \pm$ $39.8 \mu \mathrm{m} p=0.04$, respectively).

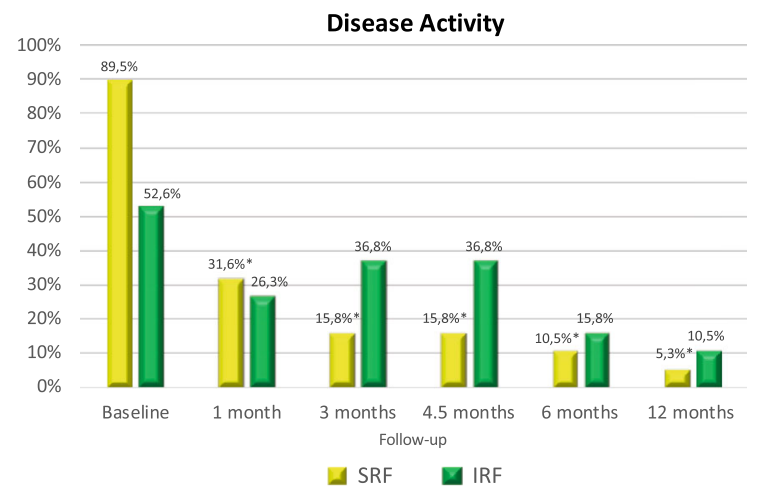

Fig. 4 Graphic of clinical data: disease activity, SRF = subretinal fluid; IRF $=$ intraretinal fluid, $* p \leq 0.05, * * p \leq 0.005$ compared with baseline 


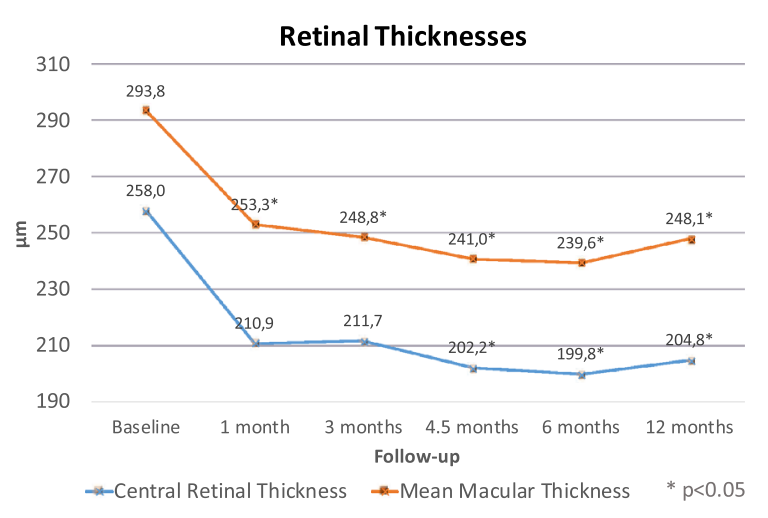

Fig. 5 Graphic of clinical data: retinal thicknesses, CRT $=$ Central Retinal Thickness; MMT $=$ Mean Macular Thickness, $* p \leq 0.05, * * p \leq 0.005$ compared with baseline

MMT was $293.8 \pm 32.1 \mu \mathrm{m}$ at baseline; it maintained significant reduction after 1, 3, 4.5, 6 and 12 months $(253.3 \pm 25.9 \mu \mathrm{m} \quad p=0.035,248.8 \pm$ $27.4 \mu \mathrm{m} \quad p=0.023, \quad 241 \pm 24.8 \mu \mathrm{m} \quad p=0.008$, $239.6 \pm 25.6 \mu \mathrm{m} p=0.003,248.1 \pm 29.6 \mu \mathrm{m} p=$ 0.017, respectively), also shown in Fig. 5 .

CCT showed significant reduction from baseline $(328.6 \pm 54.9 \mu \mathrm{m})$ after $1,3,4.5,6$ and 12 months $(290.8 \pm 55.9 \mu \mathrm{m} p=0.002,294.4 \pm 56.1 \mu \mathrm{m} p=$ $0.001,291.8 \pm 53 \mu \mathrm{m} p=0.013,293 \pm 51.7 \mu \mathrm{m}$ $p=0.025,289.8 \pm 44.6 \mu \mathrm{m} p=0.001$, respectively) (shown in Fig. 6).

MCT also showed significant reduction from baseline $(314.51 \pm 55.34 \mu \mathrm{m})$ after $1,3,4.5,6$ and 12 months $(281.6 \pm 5 \mu \mathrm{m} p=0.001,286.3 \pm 58.8$ $\mu \mathrm{m} p=0.008,289.6 \pm 54.6 \mu \mathrm{m} p=0.019,294.9 \pm$ $54.7 \mu \mathrm{m} \quad p=0.021, \quad 287.9 \pm 47.6 \mu \mathrm{m} \quad p=0.015$, respectively) (shown in Fig. 6).

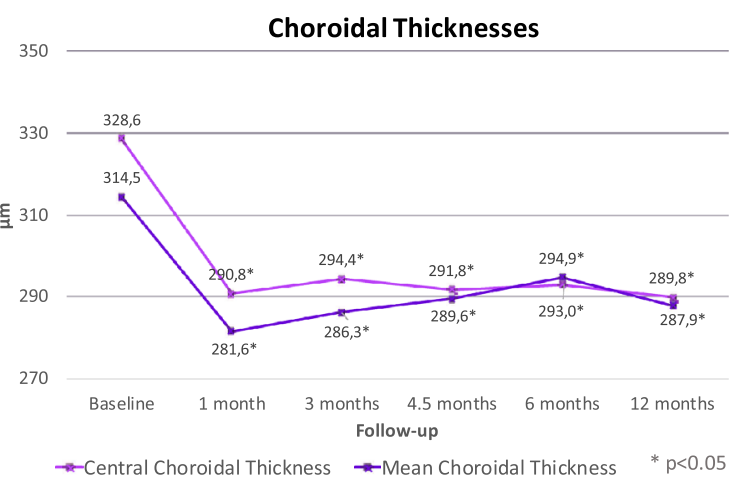

Fig. 6 Graphic of clinical data: choroidal thicknesses, CCT $=$ Central Choroidal Thickness; MCT = Mean Choroidal Thickness, $* p \leq 0.05, * * p \leq 0.005$ compared with baseline

\section{Discussion}

PCV has been described as an occult CNV since the 1990s, and it was considered to be related to AMD [13]. In 2003 Yuzawa et al. identified typical choroidal features in patients with PCV, detected by ICGA. Those choroidal vessel abnormalities were proposed to be pathogenetic of PCV [14].

In 2013 Kawamura et al. described two different patterns of PCV [9]. The first pattern was named polypoidal CNV: it showed up in FA and ICGA as a vascular network of feeder and draining vessels, new vessels growing through a break in the RPE and regular inner choroid. They also described a second pattern of PCV named typical PCV, where feeder and draining vessels were not detectable and this form of neovascularization would originate from choroidal vascularization abnormalities on atherosclerotic hyalinization.

Pang and Freund in 2015 were the first to indicate type 1 PCV neovascularization as a possible neovascular feature of pachychoroid condition, naming it pachychoroid neovasculopathy. According to these authors, pachychoroid drives a spectrum of different clinical presentations from PPE and CSC, to CNV and PCV along a continuous pathogenic evolution [1].

A thick choroid could therefore be the primum movens of a vascular condition which leads to a neoangiogenic process [5]. The dilation of vessels in choroidal Haller's layer determines choroidal compression in Sattler's layer and the thinning of choriocapillaris. Secondary hypoxic changes in RPE are responsible for epitheliopathy, serous detachment or atrophy. Manayath et co-workers showed that the coexistence of PCV and RPE changes, due to preexisting associated CSC, may support the association between these two conditions in a pathogenetic relationship with a thick choroid [5].

PCV associated with pachychoroid should therefore be differentiated from similar neovascularization associated to AMD [10, 11, 15] and it should be indicated as PPCV. Based on Gass classification, PCV is considered a type 1 subretinal neovascularization which grows underneath the RPE [16]. This anatomic location may explain the inferior response to treatment with the anti-VEGF drugs in comparison to other forms of $\mathrm{NV}$, due to an inadequate delivery of drugs to the correct site $[17,18]$. 
PCV was first treated with vPDT, which has good efficacy in closing polyps and reducing exudation, but recurrences of disease activity, i.e., exudation or hemorrhages, account for even more than $50 \%$ of cases during the first year of observation $[12,19]$.

After the introduction of anti-VEGF drugs in clinical practice, the first study comparing vPDT and anti-VEGF was the LAPTOP trial, showing a superiority of $0.5 \mathrm{mg}$ Ranibizumab compared to vPDT on naive PCV patients, in terms of visual acuity (VA) improvement [20]. The PEARL study, which focused on anti-VEGF treatment in eyes with hemorrhagic or exudative PCV, showed a low efficacy after monthly treatment with $0.5 \mathrm{mg}$ Ranibizumab, with only $31 \%$ of polypoidal complexes decreased in size after 1 year [21].

The EPIC study showed a good response to IVA labeled protocol, with a polyp closure rate of $67 \%$ in treated eyes at the 6-month endpoint. Patients included in this study had high VA at baseline and there was no statistically significant improvement in VA at the endpoint, while $90 \%$ of this group showed VA stability [22].

Although previously evaluated [23], the effectiveness of combining vPDT with intravitreal therapy (IVT) with anti-VEGF drugs in PCV treatment was stated by EVEREST II and Planet studies with evidence level I. EVEREST II demonstrated that Ranibizumab plus standard vPDT combined therapy was statistically superior in terms of VA recovery and free-from-disease interval when compared to ranibizumab monotherapy, after a 12-month follow-up. Complete polypoidal regression was also higher in the combined therapy group (69.3\% vs. 34.7\%) [24]. Choroidal response to treatment was later reported as a higher reduction in mean choroidal thickness in the combined group $(55.2 \mu \mathrm{m})$ compared to the ranibizumab monotherapy cohort $(30 \mu \mathrm{m})[25]$.

The PLANET study examined the effect of a combined therapy with Aflibercept plus vPDT compared to Aflibercept monotherapy within a 12-month follow-up. It demonstrated the non-inferiority of monotherapy treatment in comparison to the combined treatment. VA improvement after 52 weeks of follow-up was similar in both groups (10.9 vs 10.7 ETDRS letters). The closure rate of polypoidal lesions was similar in both groups (44.8 vs $38.9 \%$ ). Choroidal thickness was not analyzed. No clear information about the vPDT setting parameters, such as the dose of irradiation, fluence and spot dimension, were provided. Moreover, the combined treatment was assigned and performed after a loading dose of 3 monthly IVA, not as per protocol but only if some strict criteria were fulfilled [26].

Generally, neither the first EVEREST II report nor the PLANET study reported a basal choroidal condition or differentiated the PCV form.

In a recent study by Sakurada et coll. regarding PCV treatment, choroidal thickness was a relevant parameter to be considered together with VA, in order to evaluate the effects of vPDT + Ranibizumab and vPDT + Aflibercept combined therapies. Better VA at the endpoint was statistically associated with higher subfoveal choroidal thickness at baseline and with greater CCT reduction irrespective of treatment modalities [27]. Other studies would suggest that eyes with hyperpermeable choroid would respond better to vPDT when compared to AMD-related PCV [28] but poorly to monotherapy with anti-VEGF [11]; Yanagi et coll. addressed PCV related to pachychoroid as a subgroup of PCV, associating different clinical responses to treatment with peculiar pathogenesis and underlining the need for a lower number of IVTs when this treatment is combined with vPDT [10].

In our study, we showed the clinical results of combined vPDT + IVA therapy in patients affected by PPCV (Figs. 1 and 2).

Aflibercept was chosen due to EVEREST II and PLANET results, where monotherapy with this drug seems to be more effective than Ranibizumab monotherapy. The combined treatment rationale is based on the different targets of these therapies. The vPDT was applied for its photochemical thrombosis and remodeling of the choroidal network, acting on aneurysmal vascular origin and aneurysmal vessel closure, thus reducing choroidal congestion and hyperpermeability [12]; however, it is expected to induce overexpression of VEGF [23]. Laser spot dimension was set in order to evaluate the effect on neovascular lesions and on correspondent choroidal areas. Its effect on choroidal permeability and congestion was evaluated as a reduction in choroidal thickness and choroidal hyperpermeability [5, 10]. IVA works by blocking VEGF, neoangiogenesis and subsequently retinal exudation; its effect was evaluated as changes in retinal fluids and thicknesses.

The response to treatment was demonstrated by an improvement in all the parameters taken into account. 
CDVA progressively improved during the time of follow-up, despite relapses and IVA retreatment, as a result of recovery from chorioretinal function and resolution of retinal fluids, reaching statistical significance after 6 and 12 months. Almost all the eyes improved in CDVA after 12 months; one eye showed a relapse of activity at the 12-month follow-up visit.

IRF and SRF presence reduced as a response to anti-VEGF drug's anti-edema activity and by polypoidal closure secondary to the direct effect of PDT. Moreover, a reduction in Haller's choroidal vascular thickness and in choroidal hyperpermeability could determine a decrease of SRF and, therefore, lower stress on RPE. It is noteworthy that SRF was present in $5.3 \%$ of patients after 12 months of follow-up, and IRF in $10.5 \%$; therefore, our data showed a significant reduction in SRF in the majority of patients and a reduction in IRF as well, even though the latter was not proven to be statistically significant. Since SRF and IRF were evaluated as qualitative parameters, their clinical interpretation should be related to quantitative parameters such as CRT and MMT, which were all significantly reduced after 12 months.

CRT followed the CDVA trend, and its highest reduction was after 4.5 and 6 months. MMT reduction was statistically significant after the first month of follow-up. Its value accounts for a larger area consisting of fluids, than that included in CRT, where polypoidal lesions were mostly located: in agreement with the literature, we attributed macular thickness reduction to the synergistic action of vascular obliteration vPDT-induced on polypoidal lesions and antiVEGF-mediated fluid reabsorption.

Choroidal thicknesses (CCT and MCT) displayed a statistically significant reduction after the first month of follow-up, which stabilized during the following months. These data are directly associated with vascular structural modifications vPDT-induced, with a reduction of RPE and choriocapillaris damage caused by minor vascular congestion and vessel caliper [29]. The effect on chorioretinal structures would cause a reduction of SRF, which was related more to the stress on RPE rather than to the polypoidal activity. IVA would maintain the initial effect of vPDT, keeping the retina dry.

The number of IVA (mean 4.6 IVA/year) administered is consistent with the EVEREST II study (Ranibizumab + vPDT + PRN protocol: 5.2 IVT/ year, VS monotherapy group: 7.3 IVT/year), but not comparable to the PLANET study due to the strict year-long treatment regimen adopted (8 IVT/ year + rescue vPDT) [24-26]. Comparison with other studies is not possible due to differences in protocols, selection criteria and follow-up. In our opinion, PPCV is better controlled by combined therapy which allows for a fewer number of IVA.

A limitation of the study was the low number of patients recruited: the cohort was, however, highly selected among patients fulfilling all the pachychoroid criteria. Furthermore, no control group with monotherapy treatment was present. CDVA showed high standard deviation, due to retinal hemorrhages at the baseline but also to high VA recovery after disease activity resolution. Our hypothesis would be strongly reinforced by morphological analysis of choroidal changes, which are only indirectly proved by choroidal thicknesses. However, while ICGA only shows hyperpermeability, attempts at a quantitative analysis of choroidal vessels, as the pachychoroid index, are still not validated [30].

\section{Conclusion}

PPCV should be distinguished from other forms of PCV for its peculiar anatomical and clinical characteristics. The comprehension of its pathogenesis currently supports the choice of a combined therapy vPDT + IVT of anti-VEGF drugs. The therapeutic protocol used in this study resulted in good control of the disease and anatomic changes that support CDVA improvement. If further data confirms the role of choroidal congestion in PPCV development, preventive treatment of the pachychoroid condition could reduce the incidence of this complication.

Author Contributions Maria Vadalà: conceptualization, methodology, analysis and interpretation of data, writing original draft; Massimo Castellucci: conceptualization, methodology, investigation, analysis and interpretation of data, writing original draft; Giulia Guarrasi: investigation, analysis and interpretation of data, writing review; Giovanni Cillino: analysis and interpretation of data, writing review; Vincenza Maria Elena Bonfiglio: analysis and interpretation of data, writing review; Alessandra Casuccio: formal and statistical analysis, validation of data, writing review; Salvatore Cillino: validation of data, writing review. All authors have read and agreed to the published version of the manuscript. 
Funding This study received no funding.

Data Availability The data are only available to the researchers. They are partially stored as electronic records and preserved according to Italian privacy laws.

\section{Declarations}

Conflicts of interest Maria Vadalà is a consultant for Allergan Italia SpA, Bayer Italia Spa, Novartis Italia SpA. The other authors declare no conflict of interest. No author has a proprietary interest in this study.

Ethics approval The research was conducted ethically in accordance with the declaration of Helsinki. As stated in the manuscript, informed consent documents were obtained from all individual participants included in the study, to the data collection for the study and the scientific use of them.

Human and animal rights All procedures performed in studies involving human participants were in accordance with the ethical standards of the institutional and/or national research committee and with the 1964 Helsinki declaration and its later amendments or comparable ethical standards.

\section{References}

1. Pang CE, Freund KB (2015) Pachychoroid neovasculopathy. Retina 35(1):1-9. https://doi.org/10.1097/IAE. 0000000000000331

2. Warrow DJ, Hoang QV, Freund KB (2013) Pachychoroid pigment epitheliopathy. Retina 33(8):1659-1672. https:// doi.org/10.1097/IAE.0b013e3182953df4

3. Balaratnasingam C, Lee WK, Koizumi H, Dansingani K, Inoue M, Freund KB (2016) Polypoidal choroidal vasculopathy: a distinct disease or manifestation of many? Retina 36(1):1-8. https://doi.org/10.1097/IAE.0000000000000774

4. Iida T, Kishi S, Hagimura N, Shimizu K (1999) Persistent and bilateral choroidal vascular abnormalities in central serous chorioretinopathy. Retina 19(6):508-512

5. Manayath GJ, Shah VS, Saravanan VR, Narendran V (2018) Polypoidal choroidal vasculopathy associated with central serous chorioretinopathy: pachychoroid spectrum of diseases. Retina 38(6):1195-1204. https://doi.org/10.1097/ IAE.0000000000001665

6. Ersoz MG, Arf S, Hocaoglu M, Muslubas IS, Karacorlu M (2018) Indocyanine green angiography of pachychoroid pigment epitheliopathy. Retina 38(9):1668-1674. https:// doi.org/10.1097/IAE.000000000000177

7. Cheung CMG, Lee WK, Koizumi H, Dansingani K, Lai TY, Freund KB (2019) Pachychoroid disease. Eye 33(1):14-33. https://doi.org/10.1038/s41433-018-0158-4

8. Yannuzzi LA, Freund KB, Goldbaum M, Scassellati-Sforzolini B, Guyer DR, Spaide RF et al (2000) Polypoidal choroidal vasculopathy masquerading as central serous chorioretinopathy. Ophthalmology 107(4):767-777

9. Kawamura A, Yuzawa M, Mori R, Haruyama M, Tanaka K (2013) Indocyanine green angiographic and optical coherence tomographic findings support classification of polypoidal choroidal vasculopathy into two types. Acta Ophthalmol 91(6):e474-e481. https://doi.org/10.1111/aos. 12110

10. Yanagi Y, Ting DSW, Ng WY, Lee SY, Mathur R, Chan CM et al (2018) Choroidal vascular hyperpermeability as a predictor of treatment response for polypoidal choroidal vasculopathy. Retina 38(8):1509-1517. https://doi.org/10. 1097/IAE.0000000000001758

11. Chang YC, Cheng CK (2020) Difference between pachychoroid and non pachychoroid polypoidal choroidal vasculopathy and their response to anti-vascular endothelial growth factor therapy. Retina 40(7):1403-1411. https://doi. org/10.1097/IAE.0000000000002583

12. Cheung CMG, Lai TYY, Ruamviboonsuk P, Chen SJ, Chen Y, Freund KB et al (2018) Polypoidal choroidal vasculopathy: definition, pathogenesis, diagnosis, and management. Ophthalmology 125(5):708-724. https://doi.org/10. 1016/j.ophtha.2017.11.019

13. Yannuzzi LA, Sorenson J, Spaide RF, Lipson B (1990) Idiopathic polypoidal choroidal vasculopathy (IPCV). Retina 10(1):1-8

14. Yuzawa M, Mori R, Kawamura A (2005) The origins of polypoidal choroidal vasculopathy. $\mathrm{Br} \mathrm{J}$ Ophthalmol 89(5):602-607

15. Lai K, Zhou L, Zhong X, Huang C, Gong Y, Xu F et al (2018) Difference of choroidal vasculature between polypoidal choroidal vasculopathy and neovascular AMD on OCT: from the perspective of pachychoroid. Ophthalmic Surg Lasers Imaging Retina 49(10):e114-e121. https://doi. org/10.3928/23258160-20181002-13

16. Gass JD (1997) Stereoscopic atlas of macular diseases. CV Mosby, 4th ed. St Louis, pp. 26-30.

17. Cho M, Barbazetto IA, Freund KB (2009 Jul) Refractory neovascular age-related macular degeneration secondary to polypoidal choroidal vasculopathy. Am J Ophthalmol 148(1):70-78. https://doi.org/10.1016/j.ajo.2009.02.012

18. Stangos AN, Gandhi JS, Nair-Sahni J, Heimann H, Pournaras CJ, Harding SP (2010) Polypoidal choroidal vasculopathy masquerading as neovascular age-related macular degeneration refractory to ranibizumab. Am J Ophthalmol 150(5):666-673. https://doi.org/10.1016/j.ajo.2010.05.035

19. Palkar AH, Khetan V (2019) Polypoidal choroidal vasculopathy: An update on current management and review of literature. Taiwan J Ophthalmol. 9(2):72-92. https://doi. org/10.4103/tjo.tjo_35_18

20. Oishi A, Miyamoto N, Mandai M, Honda S, Matsouka T, Oh $\mathrm{H}$ et al (2014 May) LAPTOP study: a 24-month trial of verteporfin versus ranibizumab for polypoidal choroidal vasculopathy. Ophthalmology 121(5):1151-1152. https:// doi.org/10.1016/j.ophtha.2013.12.037

21. Kokame GT, Yeung L, Teramoto K, Lai JC, Wee R (2014) Polypoidal choroidal vasculopathy exudation and hemorrhage: results of monthly ranibizumab therapy at one year. Ophthalmologica 231(2):94-102. https://doi.org/10.1159/ 000354072

22. Kokame GT, Lai JC, Wee R, Yanagihara R, Shantha JG, Ayabe J et al (2016 Jul) Prospective clinical trial of Intravitreal aflibercept treatment for polypoidal choroidal vasculopathy with hemorrhage or exudation (EPIC study): 
6-month results. BMC Ophthalmol 27(16):127. https://doi. org/10.1186/s12886-016-0305-2

23. Wang W, He M, Zhang X (2014 Oct) Combined intravitreal anti-VEGF and photodynamic therapy versus photodynamic monotherapy for polypoidal choroidal vasculopathy: a systematic review and meta-analysis of comparative studies. PLoS ONE 9(10):e110667. https://doi.org/10.1371/ journal.pone.0110667

24. Koh A, Lai TYY, Takahashi K, Wong TY, Chen LJ, Ruamviboonsuk P et al (2017 Nov) Efficacy and safety of ranibizumab with or without verteporfin photodynamic therapy for polypoidal choroidal vasculopathy: a randomized clinical trial. JAMA Ophthalmol 135(11):1206-1213. https://doi.org/10.1001/jamaophthalmol.2017.4030

25. Lai TYGT, Margaron P, Tan C (2017) Anatomical outcomes of ranibizumab $0.5 \mathrm{mg}$ combined with verteporfin photodynamic therapy versus ranibizumab monotherapy in patients with polypoidal choroidal vasculopathy: 12-month results from the EVEREST II study. 17th EURETINA Congress; Barcelona 2017.

26. Wong TY, Ogura Y, Lee WK, Iida T, Chen SJ, Mitchell P et al (2019) Efficacy and safety of intravitreal aflibercept for polypoidal choroidal vasculopathy: two-year results of the aflibercept in polypoidal choroidal vasculopathy study. Am J Ophthalmol 204:80-89. https://doi.org/10.1016/j.ajo. 2019.02.027
27. Sakurada Y, Sugiyama A, Tanabe N, Kikushima W, Kume A, Lijima H (2017) Choroidal thickness as a prognostic factor of photodynamic therapy with aflibercept or ranibizumab for polypoidal choroidal vasculopathy. Retina 37(10):1866-1872. 0000000000001427 https://doi.org/10.1097/IAE.

28. Hata M, Tagawa M, Oishi A, Kawashima Y, Nakata I, Akagi-Kurashige Y et al (2019) Efficacy of photodynamic therapy for polypoidal choroidal vasculopathy associated with and whitout pachychoroid phenotypes. Ophthalmol Retina 3(12):1016-1025. https://doi.org/10.1016/j.oret. 2019.06.013

29. Maruko I, Iida T, Sugano Y, Saito M, Sekiryu T (2011 Apr) Subfoveal retinal and choroidal thickness after verteporfin photodynamic therapy for polypoidal choroidal vasculopathy. Am J Ophthalmol 151(4):594-603. https://doi.org/10. 1016/j.ajo.2010.10.030

30. Daizumoto E, Mitamura Y, Sano H, Akaiwa K, Niki M, Yamanaka C et al (2017) Changes of choroidal structure after intravitreal aflibercept therapy for polypoidal choroidal vasculopathy. Br J Ophthalmol 101(1):56-61. https:// doi.org/10.1136/bjophthalmol-2016-30969457

Publisher's Note Springer Nature remains neutral with regard to jurisdictional claims in published maps and institutional affiliations. 